\title{
Determination of the Li I 4d-4f Energy Separation Using Active Spectroscopy
}

\author{
K. Tsigutkin ${ }^{*}, 1$ E. Stambulchik ${ }^{1}$, Y. Maron ${ }^{1}$ and A. Tauschwitz ${ }^{2}$ \\ ${ }^{1}$ Faculty of Physics, Weizmann Institute of Science, Rehovot 76100, Israel \\ ${ }^{2}$ GSI, Darmstadt, Germany
}

Received October 3, 2004; accepted in revised form December 3, 2004

PACS numbers: 32.30. $-\mathrm{r}, 52.70 .-\mathrm{m}, 32.60 .+\mathrm{i}$

\begin{abstract}
An accurate knowledge of the LiI 4d-4f energy separation is essential for the determination of electric fields, as is pursued using several modern diagnostic techniques. However, there is a rather large spread in the values of this quantity in the available data sources. We have measured the Li I $4 \mathrm{~d}-4 \mathrm{f}$ energy separation using a technique that combines laser-induced-fluorescence with the utilization of collisional excitations. The plasma used for these measurements is laser-produced, which allows for selection of an electron-density range for which line shifts due to the plasma microfields are sufficiently small. An observation of the forbidden $2 \mathrm{p}-4 \mathrm{f}$ line provides the information on the microfields that allows for accounting for the Stark-shifts and evaluating the 4d-4f energy separation in the field-free limit. A comparison of the measured value with a few previous measurements allows for resolving the uncertainties in this quantity.
\end{abstract}

\section{Introduction}

An experimental determination of the energy separation between atomic or ionic levels is required both for examining atomic-physics calculations and the employment of numerous spectroscopic diagnostics. In particular, knowledge of the LiI $4 \mathrm{~d}-4 \mathrm{f}$ level energy separation is essential for the determination of electric fields in plasmas or neutral clouds. The mixing of these rather close levels due to electric fields results in the level shifts (the Stark effect) and appearance of dipoleforbidden transitions (e.g. 2p-4f), allowing for the determination of such fields. Indeed, such measurements have been employed in several experiments [1-5]. For relatively low electric fields $(\lesssim 20 \mathrm{kV} / \mathrm{cm})$, the $4 \mathrm{~d}-4 \mathrm{f}$ mixing coefficient is inversely proportional to the energy separation between the $4 \mathrm{~d}$ and $4 \mathrm{f}$ levels. However, as yet, the information on this energy separation is rather ambiguous. While the 4d-level energy is well known $\left(36623.4 \mathrm{~cm}^{-1}\right)$, the $4 \mathrm{f}$-level energy values quoted in tables of atomic-physics data are: $36630.2 \mathrm{~cm}^{-1}$ [6-10], $36627.4 \mathrm{~cm}^{-1}[11]$, and $36628.4 \mathrm{~cm}^{-1}[12]^{1}$. This spread in values is comparable to the $4 \mathrm{~d}-4 \mathrm{f}$ energy separation, thus this uncertainty may result in an error of factor of 2-3 in the values inferred for the electric fields.

The traditional approach for the determination of an atomiclevel energy is the measurement of the wavelength of a radiative transition between the level and another level with a well-known energy. The only dipole-allowed transition originating from the $4 \mathrm{f}$ level is the $3 \mathrm{~d}-4 \mathrm{f}$ transition. The energy of the $3 \mathrm{~d}$ level is well known $\left(31283.1 \mathrm{~cm}^{-1}\right)$ and there are no discrepancies in its value, as quoted by the different atomic data tables. These two values $\left(E_{3 d}\right.$ and $\left.\lambda_{3 d-4 f}\right)$ are sufficient to determine the $E_{4 f}$ value.

\footnotetext{
*Email address: k.tsigutkin@weizmann.ac.il

${ }^{1}$ Here and further on, we indicate a precision of $0.1 \mathrm{~cm}^{-1}$, which is more than sufficient for observing the spread in the data. Also, the spread is far beyond the quoted error-bars.
}

The main source of the uncertainties in this approach is the Stark shift of the $4 \mathrm{f}$ level due to static or dynamic electric fields present in the experimental setups, where electric fields of a few $\mathrm{kV} / \mathrm{cm}$ may cause significant uncertainties in the 4f energy determination. To the best of our knowledge, using this approach, no experimental determination of the $4 \mathrm{~d}-4 \mathrm{f}$ energy separation was made in which the effect of the possibly-present electric field on the measurement uncertainty had been accounted for.

For the first time, the $3 \mathrm{~d}-4 \mathrm{f}$ transition was observed almost a century ago by Paschen, citing the wavelength of $18697.0 \AA^{2}$ [13]. A later measurement of this transition by Johansson [14] yielded a different value for the wavelength, $18703.1 \AA$, which is quoted in two data tables $[6,11]$. Using the old Paschen data, one obtains $E_{4 f}=36630.2 \mathrm{~cm}^{-1}$. The wavelength given by Johansson results in $E_{4 f}=36628.4 \mathrm{~cm}^{-1}$. In several popular data compilations [6-10], the value of $E_{4 f}$ based on the Paschen measurement is used ${ }^{3}$. On the other hand, the Johansson measurement was used to infer the $E_{4 f}$ value by Lindgård and Nielsen for the preparation of their transition probability tables [16]. The latter are referred to by Kurucz [12]. Further, it should be mentioned that two of the data sources $[6,11]$ cite Johansson's data for $\lambda_{3 d-4 f}$, but the cited $E_{4 f}$ values are inconsistent with it.

In a more recent experimental work by Radziemski et al. [17], the $\lambda_{2 p-4 d}$ and $\lambda_{3 d-4 f}$ wavelengths were measured with a high accuracy. However, the authors note that although accurate, the values they obtain may be different from these that would be observed in field-free conditions. This results from the $4 \mathrm{~d}$ and 4f Stark shifts under the electric fields possibly occurring in their study, as pointed out in the above. For example, we believe that an electric field of at least $0.5 \mathrm{kV} / \mathrm{cm}$ may be inferred from the $3 d-5 f / 5 g$ spectrum presented in the paper. Furthermore, the $3 d-4 f$ line in that experiment could possibly be emitted from a different region with a higher field. In experiments with the hollow cathode discharge, electric fields as high as a few $\mathrm{kV} / \mathrm{cm}$ have been observed [18]. Thus, it is our belief that the error bars quoted in Ref. [17] for the value of the 4d-4f separation $\left(\Delta E_{4 d, 4 f}=4.99 \pm 0.005 \mathrm{~cm}^{-1}\right)$ do not reflect the accuracy in the field-free limit.

An alternative approach for the determination of the $4 d-4 f$ energy separation was pursued in a work by Rebhan et al. [19], where $\Delta E_{4 d, 4 f}$ was inferred from observations of the dipoleforbidden $2 \mathrm{p}-4 \mathrm{f}$ transition in the presence of an oscillating electric field. In this experiment, RF electric fields were used to stimulate emission of the $2 \mathrm{p}-4 \mathrm{f}$ forbidden satellite lines in a lithium vapor. The measurements were performed with different amplitudes of

\footnotetext{
${ }^{2}$ All wavelengths are given in air.

${ }^{3}$ It should be noted that [9] and [10] refer to an unpublished work by R. L. Kelly [15], who, apparently, used Paschen's data.
} 
the RF fields, and the $4 \mathrm{~d}-4 \mathrm{f}$ energy separation was obtained from extrapolating the results to zero-field conditions, resulting in $\Delta E_{4 d, 4 f}=5.0 \mathrm{~cm}^{-1}$, which is consistent with Johansson's and Radziemski's measurements.

The present work was motivated by the discrepancies between the different values quoted for the $4 \mathrm{f}$ level energy and by the necessity to determine this energy for both known and low enough electric fields so that a high accuracy is obtained reliably for this energy. The electric field strength in this experiment was in fact so chosen to cause an uncertainty in the value of the $4 \mathrm{f}$ level energy that is well within the instrumental error (spectral resolution) of the spectroscopic diagnostic system. In the measurements here reported, we utilized a dilute lithium plasma in which the microfields due to the plasma particles cause a weak $4 \mathrm{~d}-4 \mathrm{f}$ mixing, resulting in the observability of the $2 \mathrm{p}-4 \mathrm{f}$ forbidden transition. A simultaneous observation of both the allowed $(4 \mathrm{~d}-2 \mathrm{p})$ and forbidden transitions, for plasma parameters for which the Stark shift of the relevant levels is small, together with an independent measurement of the Doppler contribution and an accurate determination of the diagnosticsystem spectral response including its wings, allow for a direct and rather accurate determination of the $4 \mathrm{~d}-4 \mathrm{f}$ separation. In contrast to wavelength-based measurements $([13,14,17])$, the present method enables us to obtain the value with reliable uncertainties, as argued below. This was made possible due to the use of rather detailed collisional-radiative modelling and high-accuracy lineshape calculations in the data analysis.

To the best of our knowledge, no sufficiently precise calculations of the $\mathrm{Li} \mathrm{I} 4 \mathrm{~d}-4 \mathrm{f}$ separation are available as yet. Largescale calculations of this value using the multi-configuration Hartree-Fock method are currently in progress and will be reported elsewhere [20].

\section{The diagnostic method}

As said above, the basic idea of the method is to use plasma as a source of the electric fields, i.e., the plasma microfields cause the level mixing that stimulates the forbidden-line emission. For the plasma production, a pulsed laser beam that impinges on a lithium target is used. For the measurements, the laser-inducedfluorescence (LIF) technique is employed, where the $4 \mathrm{~d}$ and $4 \mathrm{f}$ level populations are increased by laser pumping. These levels cannot be efficiently pumped directly from the ground state $(2 s)$, since the $2 \mathrm{~s}-4 \mathrm{~d}$ and $2 \mathrm{~s}-4 \mathrm{f}$ transitions are dipole-forbidden. Thus, the laser emission is used to pump the $4 p$ level from the ground state, and the electron-collisional transfer in the plasma populates the $4 \mathrm{~d}$ and $4 \mathrm{f}$ levels (see Fig. 1). The simultaneous observation

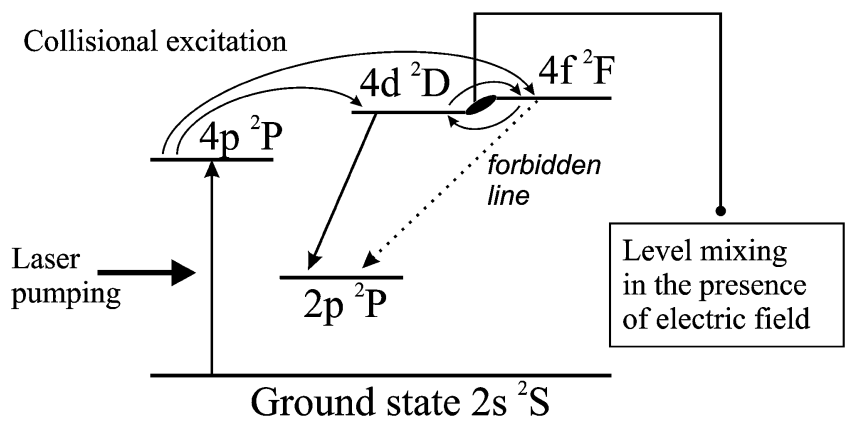

Fig. 1. The scheme of laser-driven excitations of the Li I levels. of the allowed $2 \mathrm{p}-4 \mathrm{~d}$ and the forbidden $2 \mathrm{p}-4 \mathrm{f}$ transitions allows for the $\Delta E_{4 d, 4 f}$ value to be determined.

An important feature of the present method is that the laser excitation leads to increased intensities of various lines of the excited species, due to collisional excitations and de-excitations of the atomic levels neighboring the laser-pumped level. The resulting rise in the line intensities is highly advantageous, since in addition to enhancing the intensity of the forbidden line, it allows for the observation of various lines originating from the neighboring levels. This is used to obtain simultaneous determination of both the Stark and Doppler contributions to the line shapes, which is essential for the interpretations of the line shapes, required for the determination of the energy separation. For example, a comparison of the line-shapes of the $2 \mathrm{p}-4 \mathrm{~d}$ and $2 \mathrm{p}-3 \mathrm{~d}$ transitions can reveal even a small Stark-broadening contribution to the $2 \mathrm{p}-4 \mathrm{~d}$ shape, due to the different sensitivity of the $4 d$ and $3 d$ states to the Stark effect.

The microscopic electric fields in the plasma, that are essential for the forbidden line observation, may result in level shifts that inhibit an accurate determination of the energy separation. For this reason, a plasma with a rather low density must be chosen, so that the Stark shift is too small to affect the measurement accuracy. Yet, the electron density should be high enough to allow for sufficiently high population-densities of the $4 \mathrm{~d}$ and $4 \mathrm{f}$ levels. In the laserproduced plasma plume, the density decreases with the distance from the target. By performing observations at different distances from the target, an optimal plasma density can be selected.

Furthermore, it should be noted that a high spatial resolution for the measurements is essential in order to avoid integration over a region with large variations in the plasma parameters. Since the induced fluorescence originates from the region along the pumping-laser path, observing the emitted radiation perpendicular to the beam direction allows for measurements with a high spatial resolution in 3 dimensions. Using a shortpulse pumping laser, the temporal resolution is in the range of nanoseconds.

\section{Experimental setup}

A schematic description of the experimental setup is given in Fig. 2. We produce a column of a lithium plasma by applying a 7-ns Nd:YAG laser pulse of $5 \times 10^{7} \mathrm{~W} / \mathrm{cm}^{2}$ intensity onto a solid lithium target. A dye laser is tuned to excite the LiI $4 p$ level in the plasma column from the ground state. The pumping pulse, $15 \mathrm{~ns}$ in duration, delivers intensity of $10^{6} \mathrm{~W} / \mathrm{cm}^{2}$ to the observation region. The spot size of the dye laser, which can be reduced to a sub-mm scale, determines the spatial resolution of the measurements. We scanned the plasma volume at distances between $1 \mathrm{~mm}$ and $10 \mathrm{~mm}$ from the target surface, measuring the absolute intensities and temporal evolution of the LiI $2 s-2 p$, $2 \mathrm{p}-3 \mathrm{~d}$, and $2 \mathrm{p}-4 \mathrm{~d}$ transitions. Based on these measurements and using collisional-radiative modeling [21], it was found that the electron density in this plasma region varies between $10^{14}$ and $10^{13} \mathrm{~cm}^{-3}$.

For these measurements the spectral overlap of the allowed and the forbidden emissions must be minimized. To this end, experimental conditions for which the Doppler broadening is sufficiently small had to be selected. This was achieved by selecting plasma regions along the laser-evaporated plasma column with sufficiently low LiI velocities in the direction of the observation. The lithium atoms ejected from the target surface due to the laser pulse move away from the surface with 


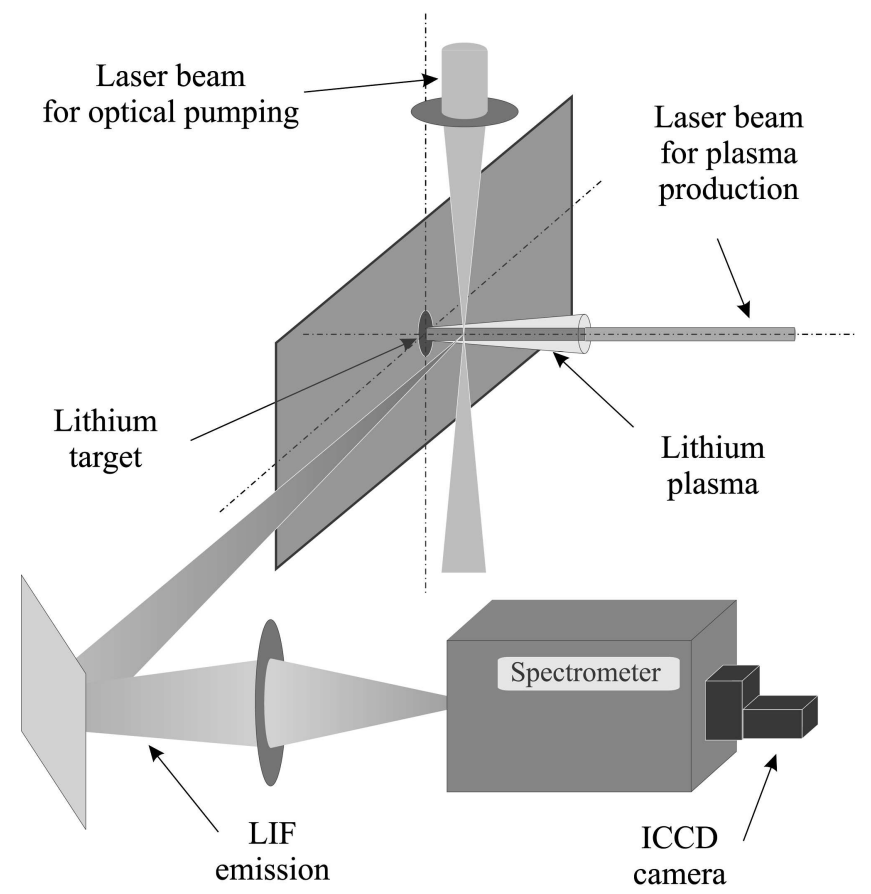

Fig. 2. Experimental setup.

velocities varying along the plasma column produced. Viewing the plasma perpendicular to the propagation direction of the plasma column, at a given distance from the target surface, allowed for selecting plasma regions with sufficiently low LiI velocities in the propagation direction by using a longer delay between the laser pulse and the measurement time (slow atoms reach the measurement region at longer delays). Generally, lower velocities in the propagation direction are correlated with lower velocities in the transverse direction, which enabled us to reach a satisfactorily low Doppler broadening in the lateral direction (the direction of observation). In the present experiment, a time delay of $450 \mathrm{~ns}$ was used, giving longitudinal and transverse velocities of $1 \times 10^{6} \mathrm{~cm} / \mathrm{s}$ and $5 \times 10^{5} \mathrm{~cm} / \mathrm{s}$, respectively.

The relatively low plasma density in these experiments resulted in a low intensity for the forbidden transition. This was due to a lower density of emitters and a small mixing of the $4 \mathrm{~d}$ and $4 \mathrm{f}$ levels in the low electric field. This necessitated the achievement of very low noise levels in the measurements and a rather accurate determination of the spectral response of the system. Such a system was constructed with a $1 \mathrm{~m}$ spectrometer with a spectral resolution down to $0.06 \AA$, and a gated (down to $5 \mathrm{~ns}$ ) intensified CCD camera, capable of recording broad spectra. The measured spectral resolution of the entire system is $0.1 \AA$, mainly determined by the spatial resolution of the ICCD camera.

\section{Results}

The spectra measured at distances of 2.5 and $5 \mathrm{~mm}$ from the target are presented in Fig. 3. In the Figure, the weak $2 p-4 f$ forbidden component is seen on both spectra. The optimal distance of the measurements from the target, in keeping with the criteria outlined in the previous sections, was found to be $5 \mathrm{~mm}$. Note that the intensity of the forbidden component is two orders of magnitude less than that of the $2 \mathrm{p}-4 \mathrm{~d}$ allowed transition. Combined with the fact that the position of the forbidden component is very close to the allowed one, this poses an additional requirement for the accuracy of the measurements and analysis of the spectral line wings. Thus, a proper account has to be taken of the effects of

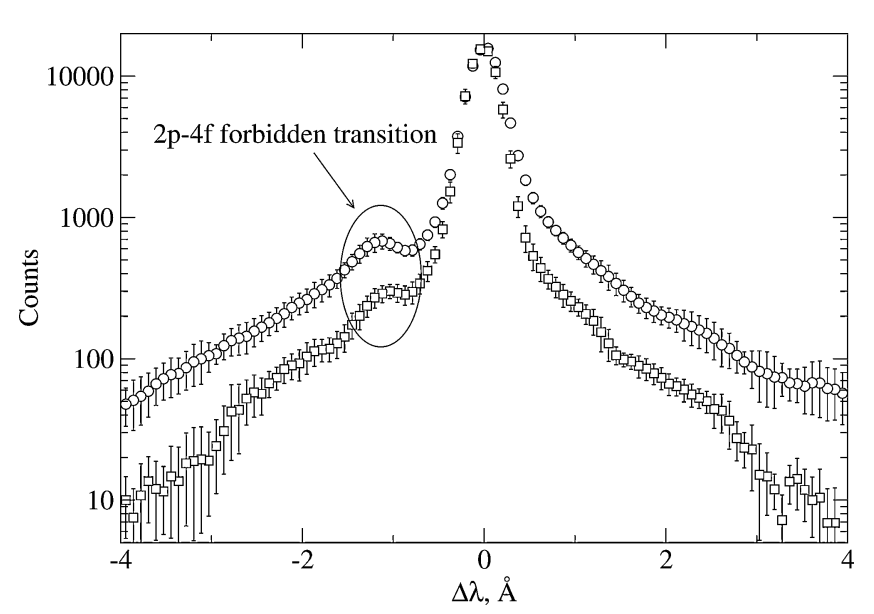

Fig. 3. The $2 \mathrm{p}-4 \mathrm{~d}$ spectra, measured at distances of $2.5 \mathrm{~mm}$ (circles) and $5 \mathrm{~mm}$ (squares) from the target.

the Doppler broadening and the instrumental spectral response function on the line shapes.

It is obvious that mechanisms leading to the allowed-line broadening also influence the forbidden line shape, which complicates the determination of the forbidden-line wavelength. In the analysis of the line shapes, three broadening mechanisms are taken into account: instrumental broadening, Doppler broadening caused by the velocity distribution of lithium atoms in the direction of the line of sight, and Stark broadening. In order to determine the exact spectral position of the forbidden line relative to the allowed one, the instrumental and Doppler broadening functions, measured separately, are deconvolved from the observed line shape, yielding the pure Stark broadened and shifted profile of the forbidden line, where the shift is too small to affect the measurement accuracy, as said above. It should be emphasized that it is this rather accurate determination of the Doppler and instrumental contributions to the line shapes that allows for a reliable determination of the difference between the wavelengths of the forbidden and allowed lines.

The instrumental response function of the spectroscopic diagnostic system was determined by measuring line spectra of $\mathrm{Ne}$ and $\mathrm{Hg}$ calibration lamps. The shapes of the $\mathrm{Hg} 4047-\AA$, Ne 6074- $\AA$, and Ne6096- $\AA$ lines are presented in Fig. 4. An important feature of the instrumental function is that it does

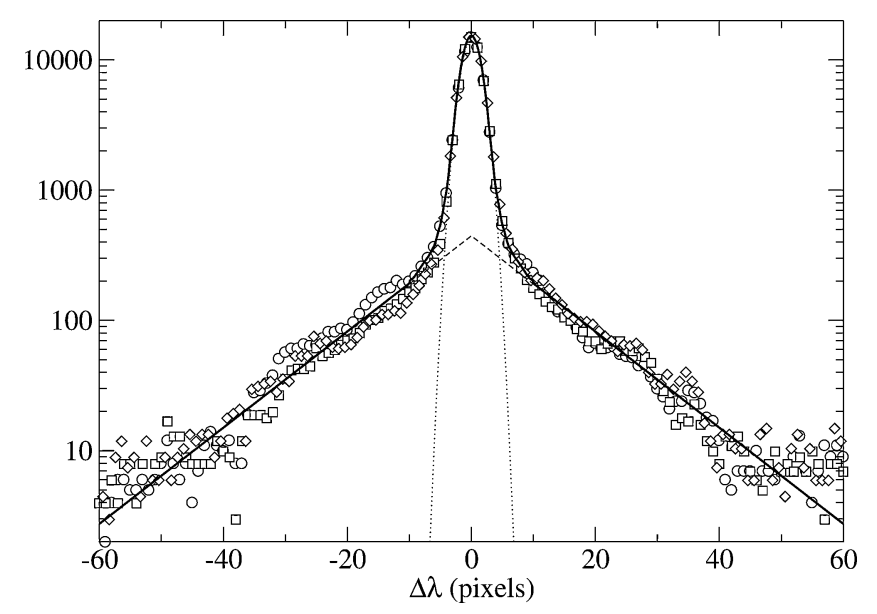

Fig. 4. The spectra of the calibration lamps: $\mathrm{Hg} 4047-\AA$ (circles), Ne 6074$\AA$ (squares), and Ne 6096- $\AA$ (diamonds). The Gaussian core and the exponential wings are depicted by the dotted and the dashed lines, respectively. The effective wavelength-independent instrumental response function is given by the solid line. 
not depend on the wavelength. Indeed, the calibration spectra almost coincide to within a single ICCD pixel, whereas $\Delta \lambda$ that corresponds to a single pixel is different for different wavelengths (for example, $0.087 \AA$ for the $\mathrm{Hg}$ line and $0.063 \AA$ for the two $\mathrm{Ne}$ lines). Therefore, it can be concluded that the main source of the instrumental broadening are the properties of the ICCD camera. This is also confirmed by the fact that the FWHM of the instrumental function significantly exceeds the quoted $0.06-\AA$ resolution of the spectrometer. From the Figure, it is seen that the core of the instrumental function is Gaussian, while the wings are nearly exponential (which is clear from the graph with the logarithmic scale, on which the wings are almost straight lines). We attribute the exponential wings to the ICCD inter-channel leak currents ${ }^{4}$. A smooth function is used for the interpolation between the Gaussian core and the exponential wings as shown by the solid curve in Fig. 4. This function is used as an effective instrumental function in the analysis of the line shapes.

The Doppler broadening is obtained from the LiI $2 \mathrm{p}-3 \mathrm{~d}$ $(6104 \AA)$ transition. The $2 \mathrm{p}-3 \mathrm{~d}$ transition is almost an order of magnitude less sensitive to the Stark broadening than the $2 \mathrm{p}-4 \mathrm{~d}$ transition, and its width, for plasma densities below $10^{14} \mathrm{~cm}^{-3}$, is only affected by the Doppler effect. Therefore, the Doppler broadening can be obtained from the $2 \mathrm{p}-3 \mathrm{~d}$ profile by deconvolving the instrumental function from the total line shape. The $2 p-3 d$ line profile is presented in Fig. 5. The instrumental function is that shown in Fig. 4, scaled based on the spectrometer dispersion $(0.083 \AA /$ pixel $)$ at this wavelength. Note that no assumption on the form of the Doppler broadening was made except for being a symmetric function. The convolution of the two broadening functions is given by the solid line, and the satisfactory fit is evident. It is interesting to note that the core of the Dopplerdominated profile is very close to Gaussian. The wings that deviate from a Gaussian can be explained by assuming that there is a fraction of very energetic Li atoms in the beam; the origin of such atoms may be charge-exchange processes.

With the instrumental and Doppler broadening at hand we can proceed to the line-wavelength analysis. The measured broadening functions are deconvolved from the total experimental line shape of the $2 \mathrm{p}-4 \mathrm{~d}$ transition in order to obtain the small Stark contribution. The Doppler broadening functions are those from Fig. 5, scaled by the $\lambda_{2 p-4 d} / \lambda_{2 p-3 d}$ factor. The resulting calculated line shapes are presented in Fig. 6. On the plot, all mechanisms (instrumental, Doppler, and Stark broadenings) contributing to the line shape are shown independently. The wavelength difference between the $2 \mathrm{p}-4 \mathrm{~d}$ and $2 \mathrm{p}-4 \mathrm{f}$ transitions is accurately determined from the Stark profile, and found to be $1.1 \pm 0.05 \AA$, corresponding to $\Delta E_{4 d, 4 f}=5.1 \pm 0.2 \mathrm{~cm}^{-1}$. This directly obtained value is in agreement with the results of Rebhan et al. [19] $\left(5.0 \pm 0.1 \mathrm{~cm}^{-1}\right)$, and with the measurements by Radziemski et al. [17], although, as was outlined in the Introduction, the latter value has an underestimated error due to the possible presence of unknown electric fields. We also note that the values of $6.8 \mathrm{~cm}^{-1}$ and $4.0 \mathrm{~cm}^{-1}$ quoted in many data

\footnotetext{
${ }^{4}$ We note that an instrumental response function that is similar in shape to our function and that is also almost independent of the wavelength, has been reported previously [22]. In that work, however, the response function was modeled by a sum of two Voigt functions. While that approach and the present one produce similarly good fits within the data error-bars, we believe the exponential wings better reflect the underlying effect that dominates the instrumental response function, which is the ICCD inter-channel leaks.
}

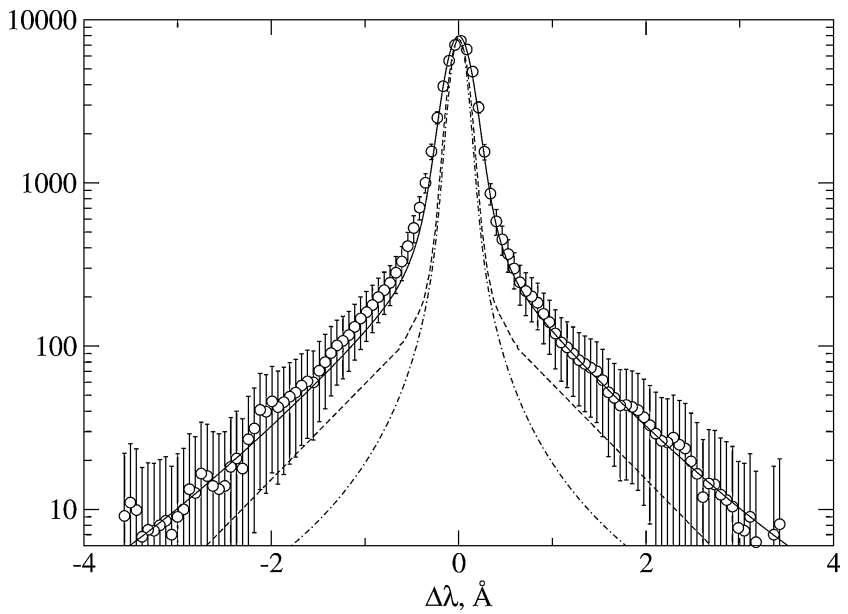

Fig. 5. Determination of the Doppler broadening using the $2 \mathrm{p}-3 \mathrm{~d}$ line shape measured at a distance of $5 \mathrm{~mm}$ from the target. The instrumental function is displayed by the dashed line, and the dot-dashed curve corresponds to the Doppler broadening.

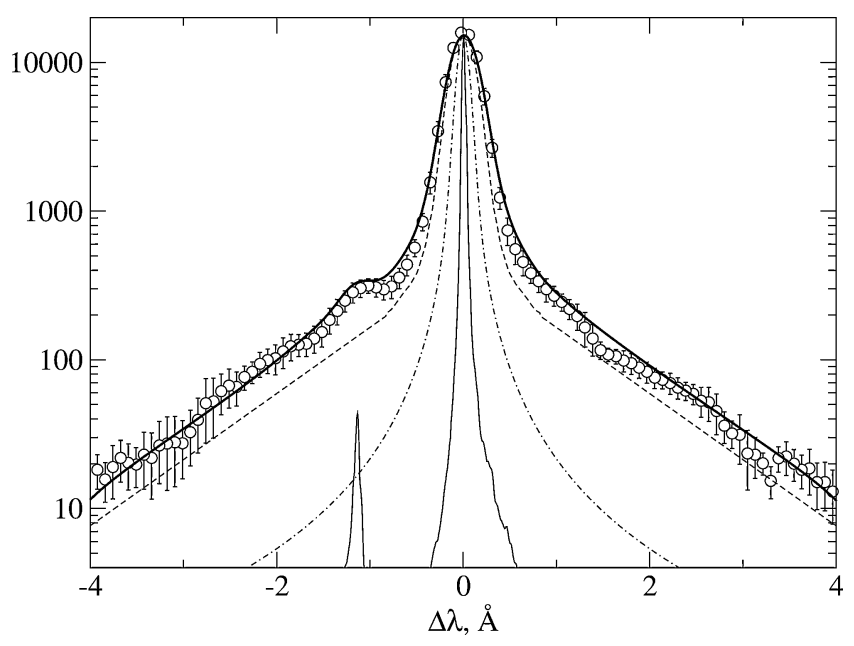

Fig. 6. Analysis of the LiI $2 \mathrm{p}-4 \mathrm{~d}$ line shape, measured at a distance of $5 \mathrm{~mm}$ from the target. The experimental data are the same as given in Fig. 3. The thin solid, dashed, and dot-dashed curves correspond to the Stark, instrumental, and Doppler broadenings, respectively. The thick solid line is a convolution of these contributions.

(Refs. [6-10] and [11], respectively), deviate significantly from the value obtained in this work.

\section{Conclusions}

The $4 \mathrm{~d}-4 \mathrm{f}$ level energy separation in lithium was measured using the observation of both the allowed $(2 \mathrm{p}-4 \mathrm{~d})$ and forbidden $(2 \mathrm{p}-$ 4f) transitions, where the latter resulted from the $4 \mathrm{~d}-4 \mathrm{f}$ level mixing due to plasma microfields. Employing laser spectroscopy, the signal-to-noise ratio achieved in the measurements was sufficiently high to allow for a reliable recording of the line profiles of both the allowed and the forbidden transitions, thus directly giving the $4 \mathrm{~d}-4 \mathrm{f}$ energy separation, found to be $5.1 \pm 0.2 \mathrm{~cm}^{-1}$.

In addition, it was demonstrated that the intensities of the $\mathrm{Li}$ I forbidden and allowed line profiles can be used for electric field measurements in transient plasmas with high temporal and spatial resolutions. Such measurements have been recently performed in an experiment employing a high-current carrying plasma and results will be published separately. 


\section{Acknowledgments}

It is our pleasure to thank V. Bernshtam for his help in performing the collisionalradiative calculations and H.-J. Kunze and Yu. Ralchenko for fruitful discussions. This work was supported by the German-Israeli Project Cooperation Foundation (DIP), Minerva Foundation (Germany), Israel Science Foundation, U.S.-Israel Binational Science Foundation, and the Meadow Fund (USA).

\section{References}

1. Sassi, M., J. Quant. Spectr. Rad. Transf. 12, 75 (1972).

2. Kawasaki, K., Usui, T. and Oda, T., J. Phys. Soc. Jpn. 51, 3666 (1982).

3. Rebhan, U., J. Phys. B 19, 3847 (1986).

4. Knyazev, B. A., Lebedev, S. V. and Mel'nikov, P. I., Sov. Phys. Tech. Phys. 36, 250 (1991).

5. Tsigutkin, K. et al., IEEJ Trans. FM 124, 501 (2004).

6. Wiese, W. L., Smith, M. W. and Glennon, B. M., "Atomic Transition Probabilities, Vol. I, Hydrogen through Neon”, Natl. Stand. Ref. Data Ser., Natl. Bur. Stand. (U.S.) 4, U.S. GPO, Washington, DC (1966).

7. Moore, C. E., "Atomic Energy Levels", Vol. I, Nat. Bur. Stand. Ref. Data Ser., Nat. Bur. Stand. (U.S.), U. S. GPO, Washington, DC (1971).
8. Bashkin, S. and Stoner, J. O., "Atomic Energy Levels and Groatian Diagrams", Vol. 1, (North Holland Publ. Col., Amsterdam, Oxford, New York 1975).

9. Reader, J. and Corliss, C. H. "Wavelengths and Transition Probabilities for Atoms and Atomic Ions", U.S. GPO, Washington (1990).

10. NIST Atomic Spectra Database, http://physics.nist.gov/cgi-bin/AtData/ main_asd.

11. Striganov, A. R. and Sventitskii, N. S., "Table of Spectral Lines of Neutral and Ionized Atoms", IFI/Plenum, NY-Washington (1968).

12. Kurucz Atomic Line Database, http://cfa-www.harvard.edu/amdata/ ampdata/kurucz23/sekur.html.

13. Paschen, F., Ann. d. Physik 27, 537 (1908).

14. Johansson, I., Arkiv Fys. 15, 169 (1959).

15. Kelly, R. L., Unpublished work.

16. Lindgàrd, A. and Nielsen, S. E., At. Data Nucl. Data Tbl. 19, 553 (1977).

17. Radziemski, L. J., Engleman, Jr., R. and Brault, J. W., Phys. Rev. A 52, 4462 (1995).

18. Takiyama, K. et al., Jap. J. App. Phys. 25, L455 (1986).

19. Rebhan, U., Weigart, N. J. and Kunze, H.-J., Phys. Lett. 85A, 228 (1981).

20. Fischer, C. F. and Ralchenko, Yu., to be published.

21. Ralchenko, Yu. V. and Maron, Y., J. Quant. Spectr. Rad. Transf. 71, 609 (2001).

22. Hörling, P. and Zastrow, K.-D., J. Quant. Spectr. Rad. Transf. 53, 585 (1995). 